\title{
Estrategias educomunicativas para la formación de comportamientos ambientales responsables en escenarios empresariales: Síntesis de la experiencia en la planta de Coca-Cola, sede Bucaramanga
}

\author{
Educommunicative strategies for the creation of responsible environmental \\ behavior in business scenarios: Summary of an experience in the Coca Cola \\ plant in Bucaramanga
}

\author{
Andrea Sánchez Flórez \\ Magister en Educación Ambiental \\ Empresa Opción Ambiental \\ Correo electrónico: gerencia@opcionambiental.com \\ Wilfredo Salinas Peñaloza \\ Magister en Educación con énfasis en Gestión y Evaluación Educativa \\ Universidad de Santander \\ Correo electrónico: wilfrestone@gmail.com
}

Recibido: 27 de enero de 2016; aceptado: 13 de marzo de 2016

\section{Cómo citar este artículo:}

Sánchez, F., y Salinas, W. (2016). Estrategias educomunicativas para la formación de comportamientos ambientales responsables en escenarios empresariales: Síntesis de la experiencia en la planta de Coca-Cola, sede Bucaramanga. Espiral, Revista de Docencia e Investigación, 6(2), 65-77.

\section{Resumen}

Tipología textual y objetivo. El siguiente artículo de investigación de la línea Educación ambiental y desarrollo sostenible, se fundamenta en el proyecto de investigación titulado "Diseño de una estrategia educomunicativa para la promoción de comportamientos ambientales en Coca-Cola, sede Bucaramanga", tiene como objetivo presentar una experiencia de exploración e intervención educativa que ha trascendido la dinámica convencional de la educación en espacios escolares y se desarrolla eficazmente en escenarios empresariales.

Metodología. El artículo sintetiza un proceso de intervención educativa desde la investigación-acción con un enfoque de estudio de caso (Martínez, 2006, p. 175), en el que se pueden identificar las fases, los procedimientos y descubrimientos, respecto de la promoción de comportamientos ambientales responsables en personas adultas, cuya función es la de ser operarios de los diferentes procesos de producción en una planta industrial como escenario de acción.

Resultados. Se exponen como resultados las transformaciones en las percepciones y la comprensión de la población participante en el proyecto, en cuanto al conocimiento de comportamientos ambientales responsables en procesos productivos, así como también la pertinencia de la estrategia educomunicativa diseñada, evaluada y aplicada para producir dichas transformaciones en el personal de planta de Coca-Cola, sede Bucaramanga.

Conclusión. Se presentan los cambios respecto de las percepciones, las conductas cotidianas que se evidenciaron en los niveles de consumo de recursos y energía en los procesos productivos y la incidencia de la estrategia en dichas transformaciones, aplicada a los trabajadores de la planta. 
Palabras clave: Comportamientos ambientales responsables, conciencia ambiental empresarial, estrategias didácticas en educación ambiental, andragogía, Educomunicación.

\section{Abstract}

Textual typology and objective. The following article of research belongs to the line named Environmental Education and sustainable development, which is based on the research project entitled "Design of an educomunicative strate $g y$ for the promotion of environmental behaviors in Coca Cola, Bucaramanga," whose objective is to introduce how an experience of exploration and educational intervention has transcended the conventional dynamics of education in school spaces and is developed effectively in business scenarios.

Methodology. The article summarizes a process of educational intervention with an action research approach with a case study methodology (Martínez, 2006, p. 175), in which the phases, procedures and discoveries can be identified regarding the advertising of responsible environmental behaviors in adults whose function is to be operators of the different production processes in an industrial plant as an action setting.

Results. The transformations in the perceptions and the understanding of the population participating in the project, regarding to the knowledge of environmental strategies which are responsible for productive processes, as well as the pertinence of the educomunicative strategy designed, evaluated and applied to produce such transformations in the staff of Coca-Cola, Bucaramanga.

Conclusions. In conclusion, the article explains the changes regarding the perceptions ande the daily behaviors that were evidenced in the levels of resource and energy consumption in the productive processes as well as the incidence of the strategy in these transformations, applied to the workers of the plant.

Keywords: Responsible environmental behavior, Environmental awareness, Didactic strategies in environmental education, anagogy, Educommunication.

\section{Introducción}

El proyecto de investigación "Diseño de una estrategia educomunicativa para la promoción de comportamientos ambientales en Coca-Cola, sede Bucaramanga" surge como una iniciativa de intervención en contextos empresariales, que tiene como propósito central, el cambio de percepción y de comportamientos ambienta- les en el personal de planta de la sede Bucaramanga.

El primer aspecto que llama la atención desde el proyecto y que orientó las acciones en este, fue la poca información sobre el uso de metodologías y estrategias de promoción de comportamientos ambientales responsables por parte del personal de producción de la planta.

Si bien la empresa contaba con una oficina de Gestión Ambiental, el nivel de conocimiento sobre los comportamientos ambientales responsables identificado en el grupo de operarios de la planta, antes de la intervención, daba cuenta de una baja fundamentación y poca aplicación de los principios de seguridad ambiental prescritos en la normativa nacional en el artículo $1^{\circ}$ de la Ley 1549 del 05 de julio de 2012.

La estrategia se construyó con la participación de un grupo de treinta operarios de la planta de producción, quienes compartieron en un primer momento sus percepciones sobre lo que comprendían respecto de comportamientos ambientales responsables (Álvarez \& Vega, 2009, p. 245). De allí, el reto consistió en desarrollar una estrategia que facilitara el acceso a la información sobre las diferentes acciones cotidianas que constituían riesgos ambientales y cuyo origen debía identificarse desde la observación del contexto productivo.

La intervención propuesta para asumir esta exploración, resulta del reconocimiento de los factores incidentales o indicadores desde los cuales se valoró la apropiación y práctica de comportamientos ambientales responsables por parte de los operarios, y desde este reconocimiento se pudo establecer en el inicio de la intervención, que había un manejo incipiente de la información sobre estos comportamientos, que hacían difíciles unas prácticas productivas consecuentes con dichos comportamientos ambientales. 


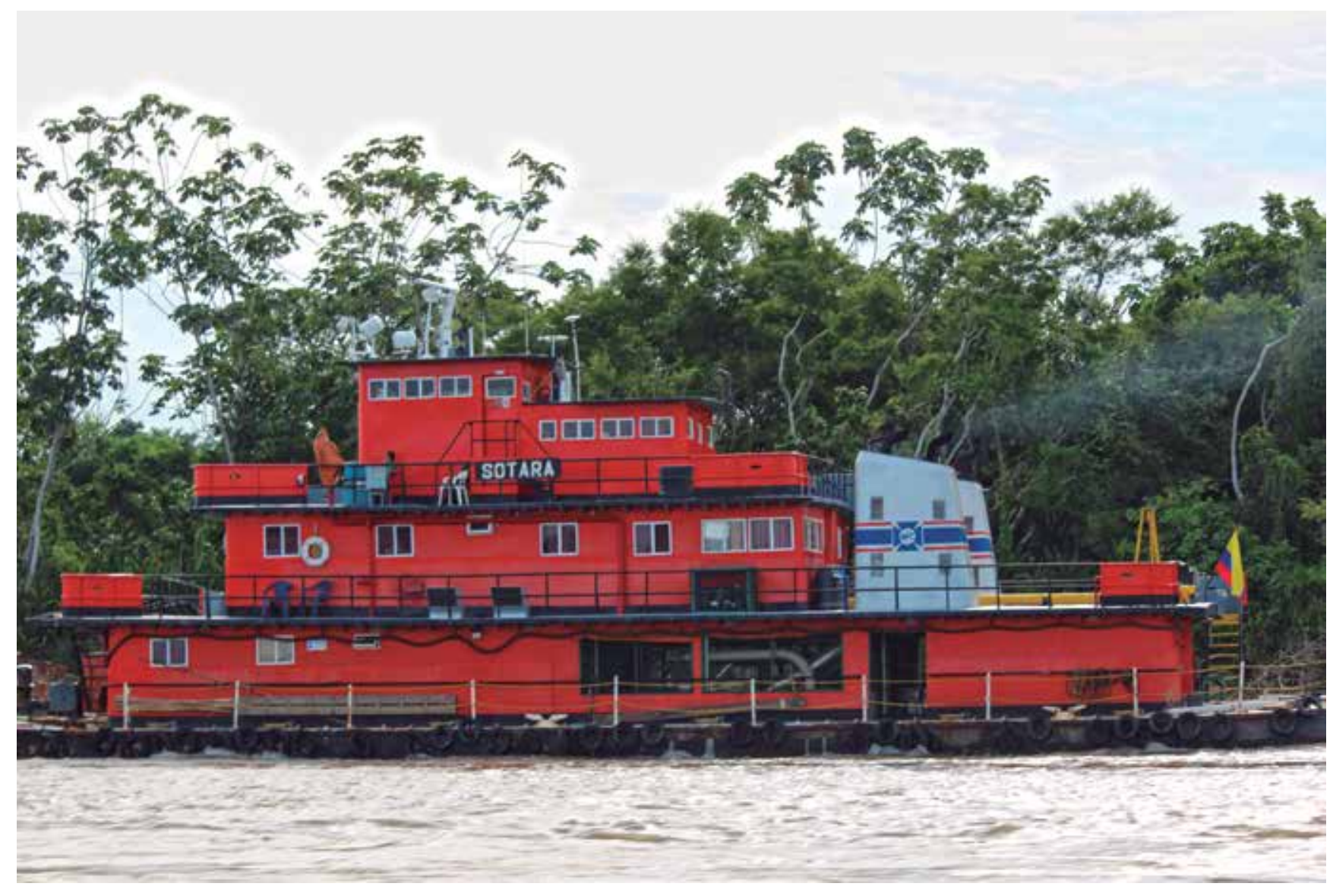

La estrategia desarrollada e implementada contó con pautas de diseño desde las cuales se integraron recursos multimedia como videos, interactividades y trabajo de campo dentro de la planta que permitiría cambios en los tipos de respuesta en el grupo de operarios y al mismo tiempo, generaría espacios de reflexión que finalmente se podrían registrar como resultados favorables en el proyecto.

Veremos a continuación cómo se produjeron los resultados del proceso de intervención y qué aportes hace este proyecto al estudio de estrategias educomunicativas de formación aplicadas en contextos empresariales.

\section{Metodología}

En la empresa Coca-Cola, sede Bucaramanga se buscó investigar y dar respuesta a la pregunta ¿De qué manera un diseño de estrategias didácticas favorecerá los comportamientos am- bientales responsables de los empleados de la planta de producción de la empresa Coca-Cola, sede Bucaramanga?

A fin de resolver el problema, se hizo un trabajo de investigación basado en la metodología cualitativa (Bonilla \& Rodríguez, 2010, p. $120)$ de tipo evaluativa (Correa, Puerta $\&$ Restrepo, 2002, pp. 17-49) y con enfoque de estudio de caso, para comprender si el comportamiento ambiental no responsable del personal de producción estaba relacionado con el rechazo al cumplimiento de las normas, la falta de mayor claridad en los nuevos conceptos o si la estrategia comunicativa y metodológica utilizada para la formación era o no efectiva para dicho propósito.

Para encontrar la respuesta se hizo recopilación de la información, utilizando como técnicas la observación estructurada no participante que se desarrolló desde una matriz categorial 
de factores que se explican en la metodología, una encuesta de respuesta abierta para identificar comportamientos y tendencias (responsables o no responsables) en dichos comportamientos, y la escala de autoevaluación desde la cual pudieron reconocer sus propias actitudes frente al compromiso del uso responsable de los recursos en el proceso productivo, que se aplicaron desde el mes de agosto hasta noviembre de 2015 .

Con la observación estructurada no participante, categorizados en cinco indicadores (energía, agua, residuos, sustancias químicas y política ambiental empresarial) de los cuales se derivaron veintiún ítems de pregunta, la investigación se centró en los aspectos relevantes que generaron patrones de comportamiento y que ayudaron a formular un nuevo diseño para la estrategia educomunicativa (Barbas, 2012, p. 174).

Como apoyo se utilizó la encuesta de respuesta abierta que contenía cinco preguntas, a fin de obtener la información directa del trabajador y conocer su punto de vista en ítems claves como la comprensión de los conceptos e identificación de los comportamientos ambientales no responsables por parte de sus compañeros y la entidad como ente administrativo. También, ver la asistencia a capacitaciones durante el tiempo laborado en el área de producción y las falencias a la hora de implementar una acción en beneficio del ambiente.

A modo de cierre se aplicó la escala de autoevaluación de manera individual a los participantes, estructurada en un formato impreso con trece (13) indicadores para reflexionar sobre el aprendizaje transmitido y detectar si hubo avances en los comportamientos ambientales responsables o si generó mayor confusión en el trabajador. Esto con el fin de con- cluir si la estrategia utilizada fue pertinente o si al contrario, se requeriría de una nueva para este caso en particular.

La población y muestra seleccionada para el estudio fueron treinta personas que laboran en el área de producción de Coca-Cola, sede Bucaramanga, área que genera el mayor consumo y afectación a los recursos naturales por su carácter productivo. Las características de las personas que participaron en la investigación fueron un $100 \%$ de sexo masculino con un nivel académico de bachiller y técnico. Sus edades oscilan entre 20 y 65 años: siendo menores de 40 años 7 personas y mayores a este rango 23 personas, por tanto, la estrategia se orientó hacia la andragogía.

\section{Resultados}

Al hacer recopilación, análisis y sistematización de la información se presentan los hallazgos en tres etapas: la primera muestra los datos registrados antes de la implementación de la estrategia educomunicativa, la segunda diseña la estrategia educomunicativa y la tercera refleja los alcances una vez se implementa el proceso formativo.

\section{Primera etapa}

Esta primera etapa involucra la observación estructurada no participante y la encuesta de respuesta abierta. La primera se aplicó en seis oportunidades con seguimientos semanales. La segunda se hizo en una única jornada dentro de las instalaciones de la entidad con un período de tiempo de 10 a 15 minutos por persona.

\section{Sobre la observación estructurada}

Es importante destacar que las veintiuna apreciaciones se organizaron en cinco categorías antes descritas, arrojando resultados que se presentan en la figura 1: 
Figura 1. Resultados de la observación estructurada no participante

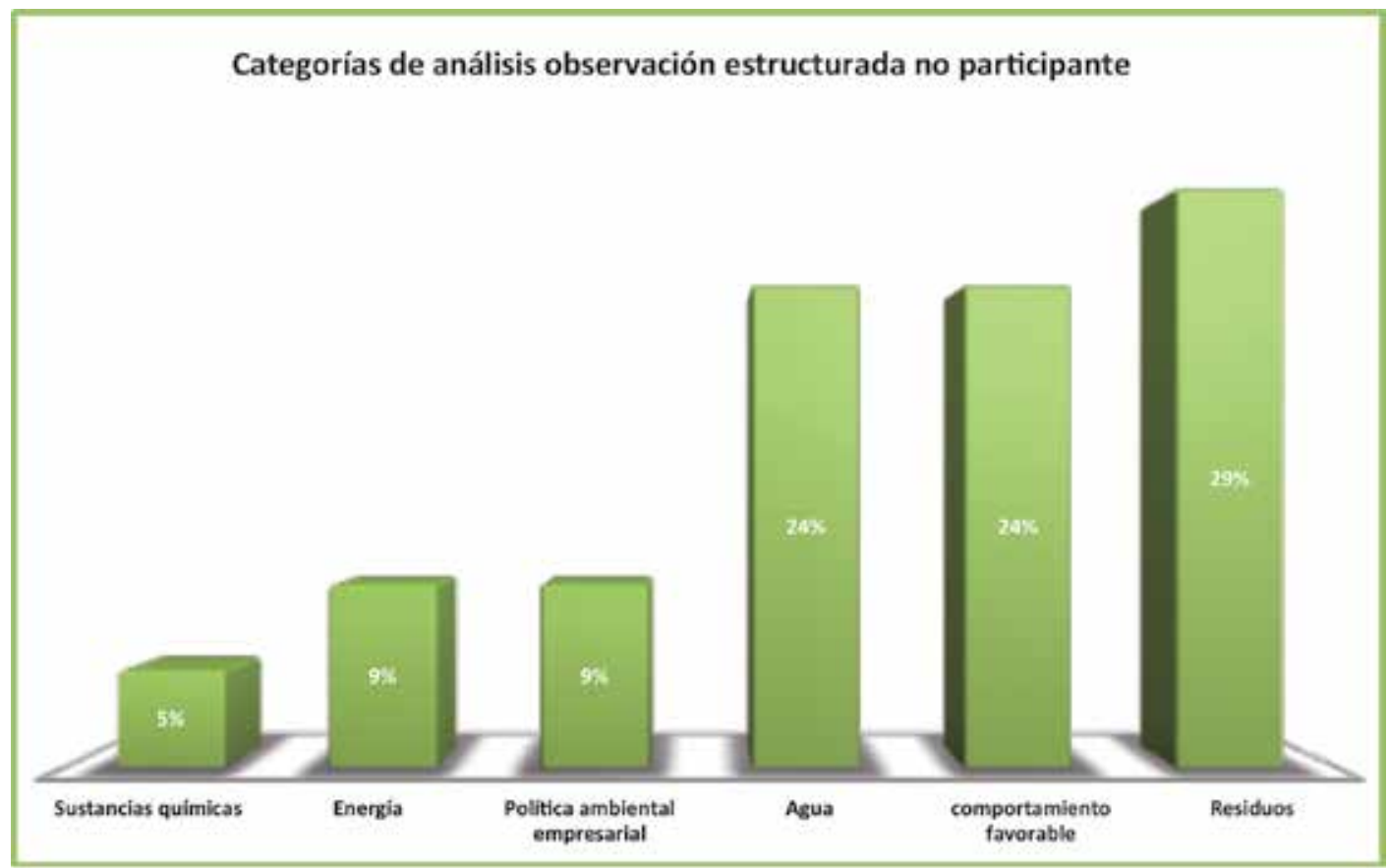

Fuente: autores.

Los procesos de producción que requirieron mayor atención fueron el manejo de residuos sólidos que obtuvo un $29 \%$, seguidos por el uso eficiente del agua con $24 \%$, y los que tuvieron menor impacto fueron el uso eficiente de la energía y la divulgación de la política ambiental empresarial, cada uno con $9 \%$, y en último lugar el manejo de las sustancias químicas con un 5\%, análisis que sirvió de referente para plantear la estrategia priorizando los aspectos de mayor representatividad como comportamientos ambientales no responsables que necesitarían una intervención educomunicativa.

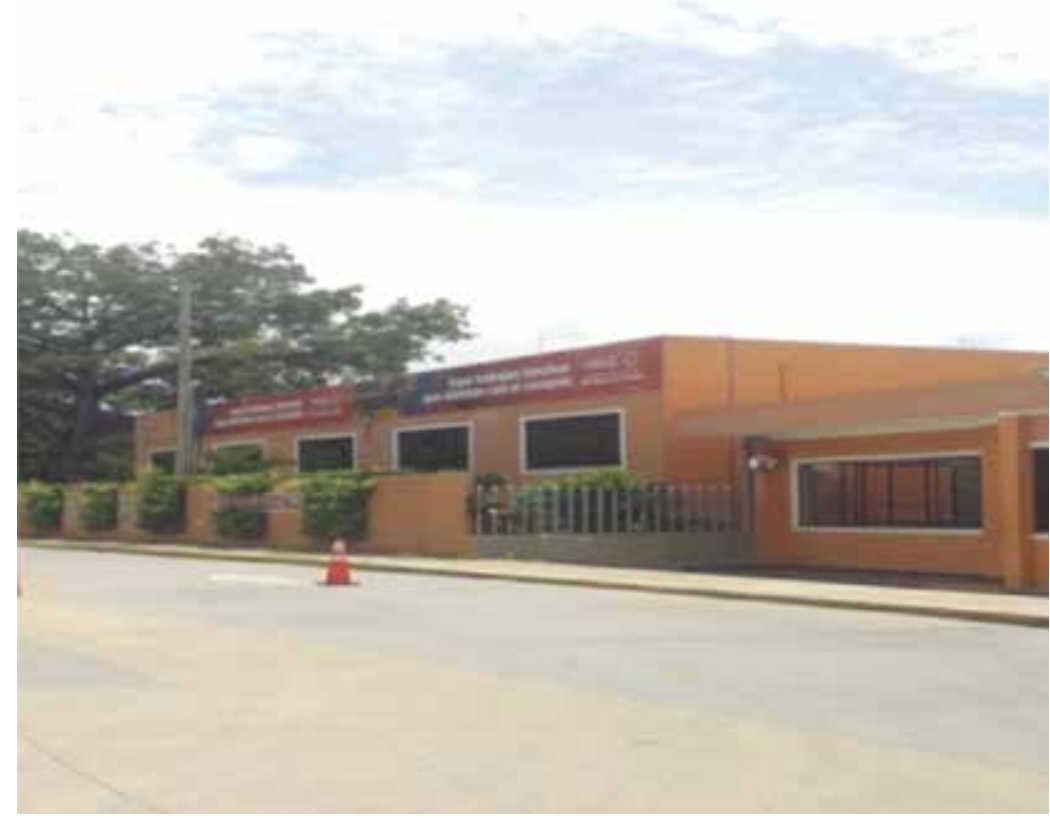

Fachada de la Planta de producción, sede Bucaramanga. 
Desde la implementación de la estrategia los comportamientos se tornaron favorables al observar que en el área dentro de la cual se hizo el análisis, se realizaron arreglos locativos e implementación de manuales. Se identificó que para el trabajador hay una relación directa entre el manejo adecuado de las sustancias químicas y el daño a la salud, por tanto, ejecuta con mayor prudencia los lineamientos para el manejo, almacenamiento y disposición final de estas sustancias y sus residuos.

La observación también reflejó que el personal mejora sus comportamientos ambientales con la presencia de un jefe inmediato o autoridad dentro de la zona de producción. Esto demuestra que el operario conoce el concepto, pero necesita reforzar sus conocimientos y la importancia de aplicarlos.

\section{Sobre la encuesta de respuesta abierta}

El personal encuestado manifiesta que aplica comportamientos ambientales responsables en diversas zonas donde se encuentre, no solo dentro de las instalaciones de la empresa, sino que procuran crear hábitos que involucren todos los espacios. Pero, también expresan la necesidad de conocer más a fondo sobre el tema en cuestión, debido a que sienten dudas a la hora de realizar una acción, al considerar que su conocimiento no es muy claro.

Por otra parte, a la pregunta ¿Qué comportamientos ambientales por parte de la empresa usted considera que son inadecuados? Se observó que de las treinta personas encuestadas, quince coinciden en afirmar que hace falta reforzar el uso eficiente del agua y diez manifiestan que lo más importante es mejorar el programa de residuos (figura 2 ).

Figura 2. Comportamientos ambientales inadecuados del área administrativa

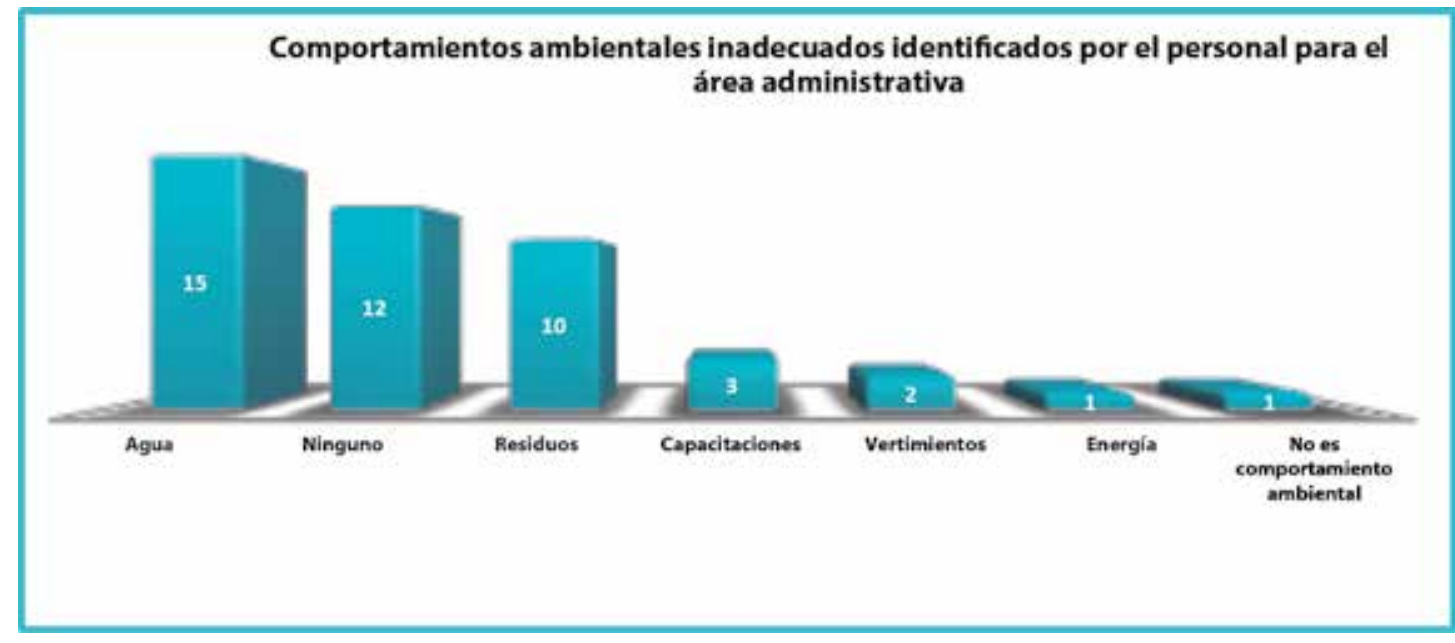

Fuente: autores.

Así también, doce consideran que aun cuando hay pequeñas mejoras por hacer a nivel general dentro de la entidad, hay un equilibrio entre el uso de los recursos naturales y la producción.

Con la pregunta: ¿Considera que en el área de producción existen inadecuados comportamientos ambientales por parte de sus com- pañeros de trabajo? Se pudo determinar que el personal de producción puede identificar que hay falencias representativas en los programas de residuos, agua y energía y que requieren de mayor capacitación para mitigar el impacto paisajístico y ambiental que se pueda ocasionar (figura 3). 
Figura 3. Comportamientos ambientales inadecuados del área de producción

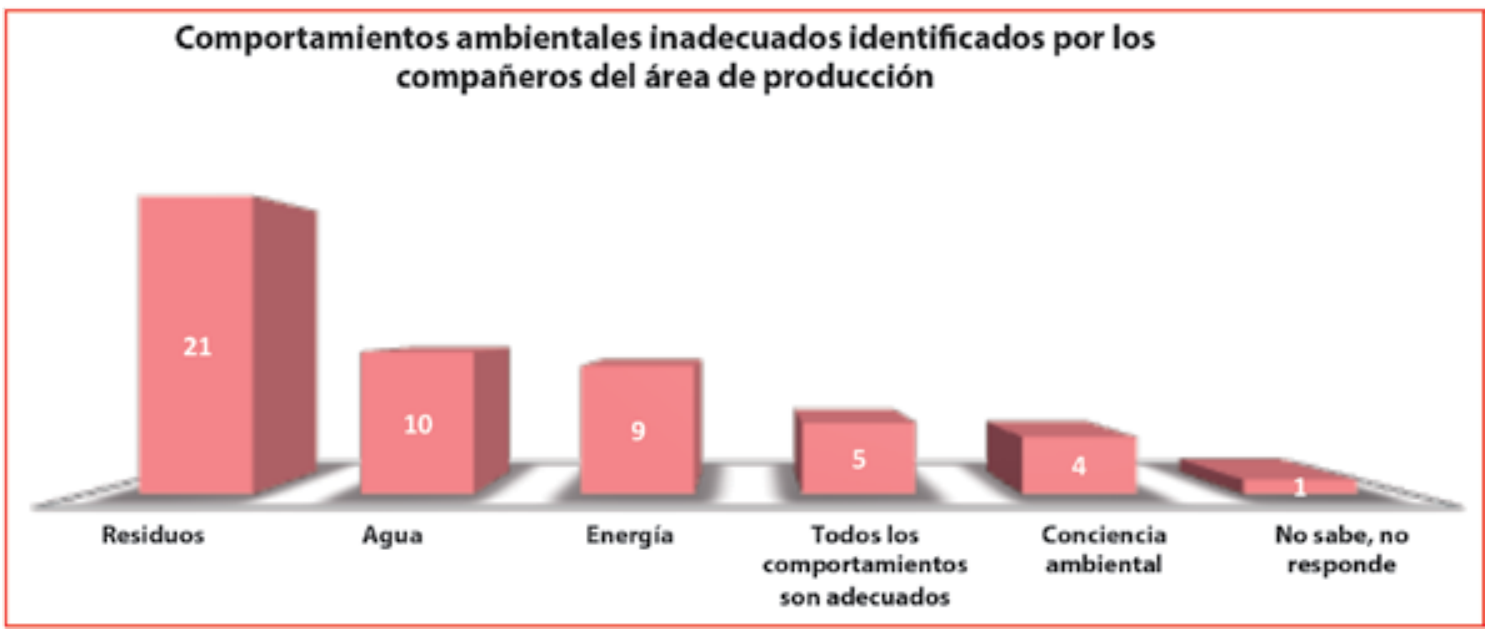

Fuente: autores.

Con la información recopilada se pudo identificar las prácticas ambientales por mejorar (tabla 1) con la estrategia educomunicativa.

Tabla 1. Prácticas ambientales por mejorar con la estrategia educomunicativa

\begin{tabular}{|c|c|}
\hline Categoría & $\begin{array}{c}\text { Prácticas ambientales por reforzar mediante el uso de } \\
\text { una estrategia educomunicativa }\end{array}$ \\
\hline Energía & $\begin{array}{l}\text { - Apagar luces del área de producción una vez finalizada } \\
\text { la jornada. } \\
\text { - Apagar equipos (maquinaria) de producción una vez } \\
\text { finalizada la producción. }\end{array}$ \\
\hline Agua & $\begin{array}{l}\text { - Cerrar el grifo mientras se enjabona o lava los dientes. } \\
\text { - Reportar fugas de agua. } \\
\text { - Manipulación adecuada de las válvulas de agua en el } \\
\text { área de producción. } \\
\text { - Uso adecuado del agua para el aseo. }\end{array}$ \\
\hline Residuos & $\begin{array}{l}\text { - No arrojar residuos u otros elementos al piso. } \\
\text { - No consumir alimentos en la zona de producción. } \\
\text { - Disminución del uso de desechables para tintos y } \\
\text { aromáticas. } \\
\text { - Clasificación adecuada en la fuente. } \\
\text { - Traslado de residuos al punto de acopio respetando la } \\
\text { clasificación en la fuente. }\end{array}$ \\
\hline Sustancias químicas & $\begin{array}{l}\text { - Utilizar la totalidad de los productos para la elaboración } \\
\text { de la gaseosa (jarabes). }\end{array}$ \\
\hline Política ambiental empresarial & $\begin{array}{l}\text { - Prestar atención a la cartelera e informes ambientales } \\
\text { suministrados por el D.G.A. } \\
\text { - Atender las sugerencias y recomendaciones que se } \\
\text { hacen por medio de la pantalla informativa (televisor). } \\
\text { - Liderazgo ambiental. }\end{array}$ \\
\hline
\end{tabular}




\section{Segunda etapa}

Teniendo el listado de los comportamientos ambientales por mejorar, se procedió a diseñar la estrategia educomunicativa pertinente para el estudio.

A fin de mejorar las acciones, mitigar el impacto a los recursos naturales y dar cumplimiento a la política ambiental empresarial, se consideró pertinente utilizar la estrategia de enseñanza aprendizaje basado en problemas $A P B$ para que el personal capacitado tuviera la oportunidad de conocer los errores que están cometiendo, el problema que están ocasionando y cómo un comportamiento ambiental responsable lo puede mitigar.

La estrategia educomunicativa aplicada tuvo por nombre "todo suma o todo resta" con el objeto de demostrar que toda acción que se realiza repercute a pequeña o gran escala en el ciclo de la naturaleza que de forma directa e indirecta también influye en la calidad de vida de los individuos, principalmente los más cercanos al área de afectación. Conviene destacar que se tuvo en cuenta no solo el nivel de formación de los operarios, sino también los factores que pudieran significar un condicionamiento para el aprendizaje de comportamientos ambientales responsables, visto desde la perspectiva andragógica.

Desde esta perspectiva de formación, era claro que el manejo de la información sobre dichos comportamientos debía permitirles un aprendizaje desde la práctica, y para ello, los referentes asociados en las capacitaciones y la intervención en el área de producción, se hizo pensando en sus funciones principalmente, que puso a prueba el método de acción o actividad (Santo Domingo, 2006, p. 7), desde el cual se establecían comportamientos ambientales responsables desde las prácticas cotidianas (manejo de procesos productivos teniendo en cuenta pautas del uso regulado del agua, la energía, los residuos, entre otros).

Los recursos educativos utilizados fueron en primera instancia las carteleras y los videos informativos como fase de fundamentación; seguidamente, desde juegos de rol y dinámicas de grupo, en cada visita de capacitación al grupo se hizo la sensibilización para prepararlo para la reflexión sobre los cambios que se esperaban en los comportamientos ambientales responsables; finalmente, se implementaron sesiones de trabajo con material documental de revistas y periódicos, que sirvieron de contexto para generar compromisos éticos en los participantes desde los cuales fueran más efectivos los cambios en el personal de la planta.

\section{Tercera etapa}

En la tercera etapa se quiso valorar el alcance que tuvo la estrategia educomunicativa "todo suma o todo resta" en el personal de producción de Coca-Cola, sede Bucaramanga, por tanto, se procedió a aplicar los instrumentos observación estructurada no participante y la escala de autoevaluación.

\section{Sobre la observación estructurada después de la estrategia}

Al aplicar nuevamente el instrumento se observó que los resultados alcanzados son favorables (figura 4). 
Figura 4. Comparativo antes y después de la estrategia

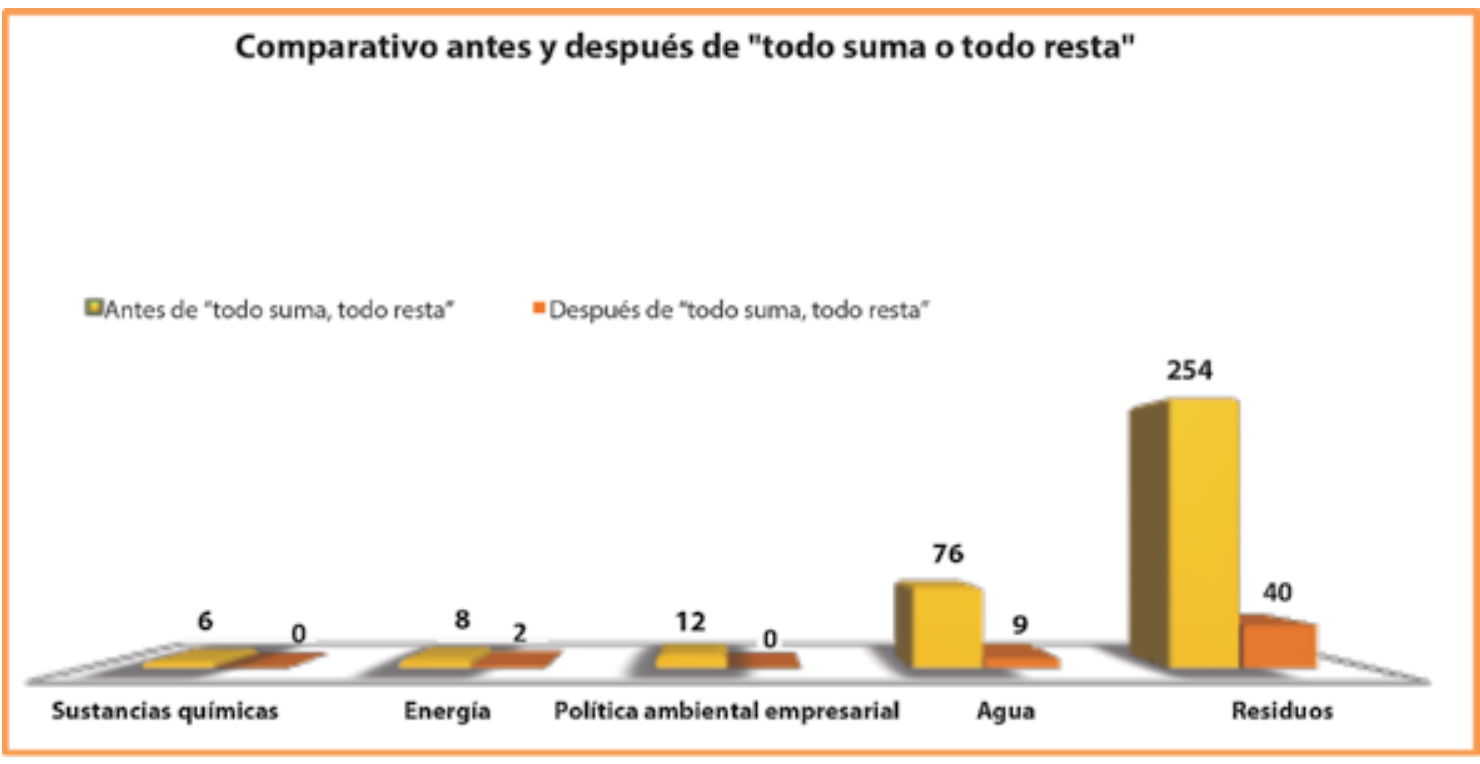

Fuente: autores.

Se identificó que de los 356 comportamientos ambientales no responsables observados en la primera etapa, solo 51 se mantienen, esto quiere decir que hasta en un $85,7 \%$ el personal de producción pudo mejorar sus acciones con la aplicación de la estrategia.

Expresado de otra manera, se evidencia que de los 59 comportamientos ambientales inadecuados registrados diariamente se pudieron reducir a 9 con la aplicación de la estrategia educomunicativa.

\section{Sobre la escala de autoevaluación}

La aplicación de la escala de autoevaluación arrojó resultados positivos. Estos, al compararlos con los obtenidos en la observación estruc- turada, confirman la aceptación por parte del personal de producción de Coca -Cola, sede Bucaramanga. Así, se percibe en los sujetos investigados el deseo de promocionar comportamientos ambientales responsables desde una percepción de responsabilidad individual y protección colectiva.

La herramienta estaba compuesta por trece preguntas que describían los comportamientos ambientales no responsables que con antelación se habían identificado. Cada pregunta presentaba una escala de 1 a 3, donde 1 es nunca, 2 es varias veces por semana y 3 es siempre (figura 5): 
Figura 5. Resultados de la escala de autoevaluación

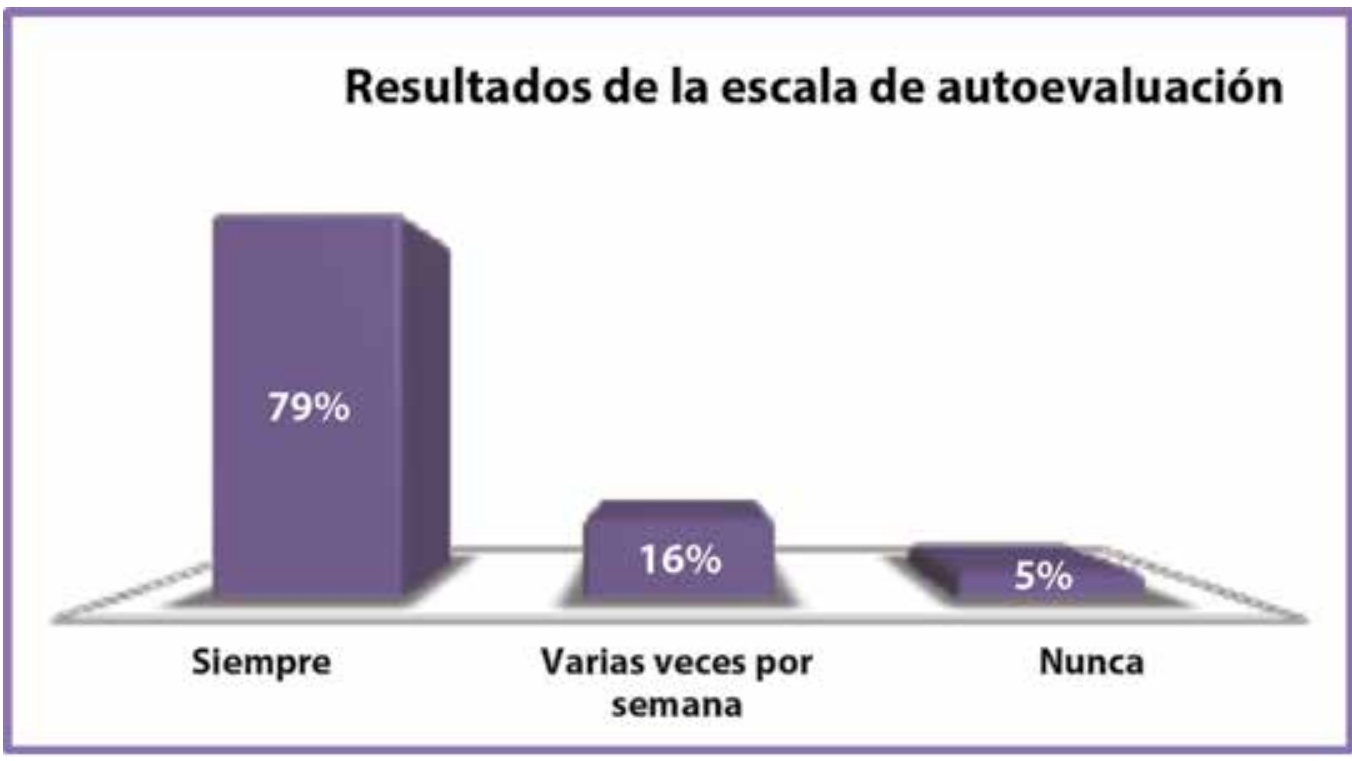

Fuente: autores.

El personal capacitado mantuvo una conducta positiva hacia el conocimiento, queriendo aprender más de los recursos naturales que los rodean y de los impactos que pueden ocasionar con sus acciones. Demostrando en los resultados que hasta en un $79 \%$ de las veces quieren mantener siempre una conducta adecuada hacia el ambiente.

El $21 \%$ restante manifiesta tener comportamientos ambientales responsables, siempre y cuando dependa de su actuar y no de otros. Es decir, no desean reportar fugas o daños ocasionados en el área.

Esto sucede debido a que manifiestan que al notificar la información no se tiene en cuenta por el área encargada y no se realiza la acción requerida.

\section{Conclusiones}

El principal aspecto que se tuvo en cuenta en la valoración de los resultados y el punto de llegada del proyecto es que la estrategia educomunicativa se desarrolló para un entor- no laboral que exigió cambios sustanciales en la mirada del proceso formativo, por lo que se trató de educación para adultos en contextos laborales u organizacionales.

Aquí, la determinación de las transformaciones de los sujetos que hicieron parte del grupo muestra del proceso se pudieron establecer desde los resultados en la evaluación final de la estrategia. Los cambios de los operarios - protagonistas del proceso- hacia la práctica de comportamientos ambientales responsables se demostró luego de que, ante la verificación final de la implementación se pudo identificar una reducción sustancial en el manejo inadecuado de los residuos discriminados en las cinco categorías preestablecidas desde el inicio (energía, agua, residuos, sustancias químicas y política ambiental empresarial), como lo demuestra la figura 4.

Seguidamente, se pudo identificar no solo las tipologías de prácticas ambientales que requerían el uso de estrategias didácticas para el apoyo de la gestión ambiental en la Empresa, sino también las formas en que se podrían fun- 
damentar los operarios en acciones correctivas para enfrentar estas problemáticas y optimizar su desempeño laboral desde un enfoque más amable y consciente de su responsabilidad en la gestión ambiental.

Se logró del mismo modo el diseño de recursos y actividades que fundamentaron las estrategias didácticas en dicha fundamentación por parte de los operarios participantes.

Como parte de dichas estrategias se diseñaron recursos con un nivel de interactividad multimedia, que fue el resultado del trabajo creativo que permitió el proceso de fundamentación de los operarios en las conductas ambientales responsables.
De otra parte, la implementación de la estrategia educomunicativa desarrollada en el marco del proyecto permitió identificar el alcance del material y los procedimientos de capacitación y actualización desarrollados desde la estrategia propuesta, con un enfoque formativo andragógico (Santo Domingo, 2006, p. 6), desde el cual se abordó el compromiso de formar al personal de planta cuyo rango de edad oscilaba entre los 40 y los 65 años de edad. Lo anterior representó una exigencia en cuanto al diseño del material audiovisual resultante, que exigía en su componente comunicativo un lenguaje común que permitiera emitir mensajes claros y pautas concretas para la acción, que luego derivaron en materiales interactivos para su consulta y aplicabilidad en escenarios de actualización permanentes dentro de la Empresa.

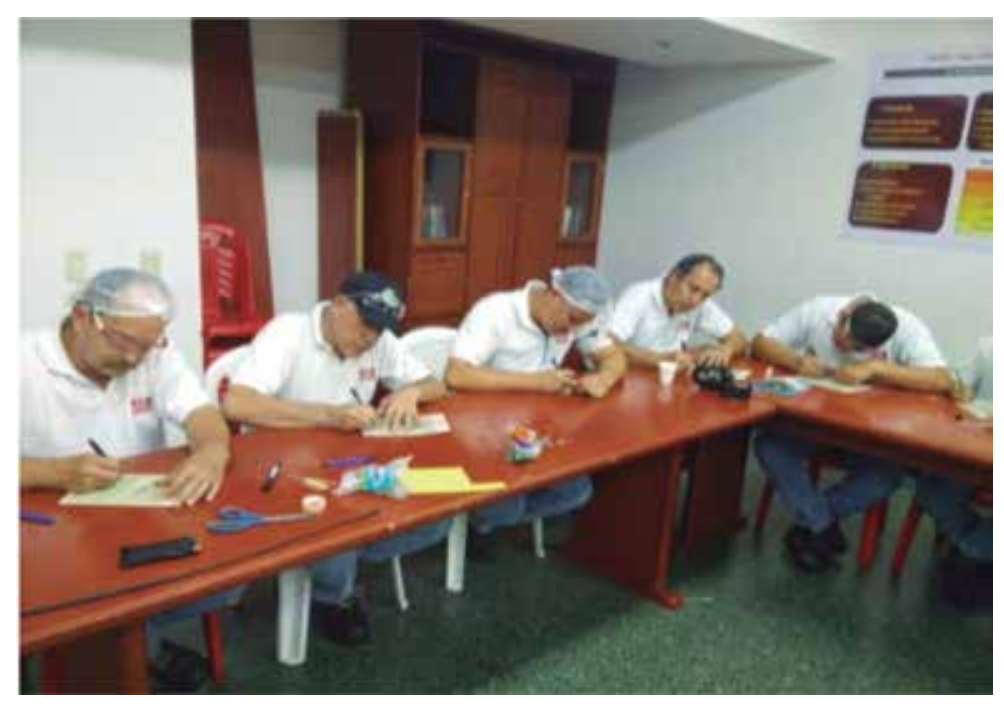

Taller realizado con los operarios de la Planta.

Fuente: autores. 
Figura 6. Aspecto de la página de inicio del objeto de aprendizaje

"Todo suma o todo resta"

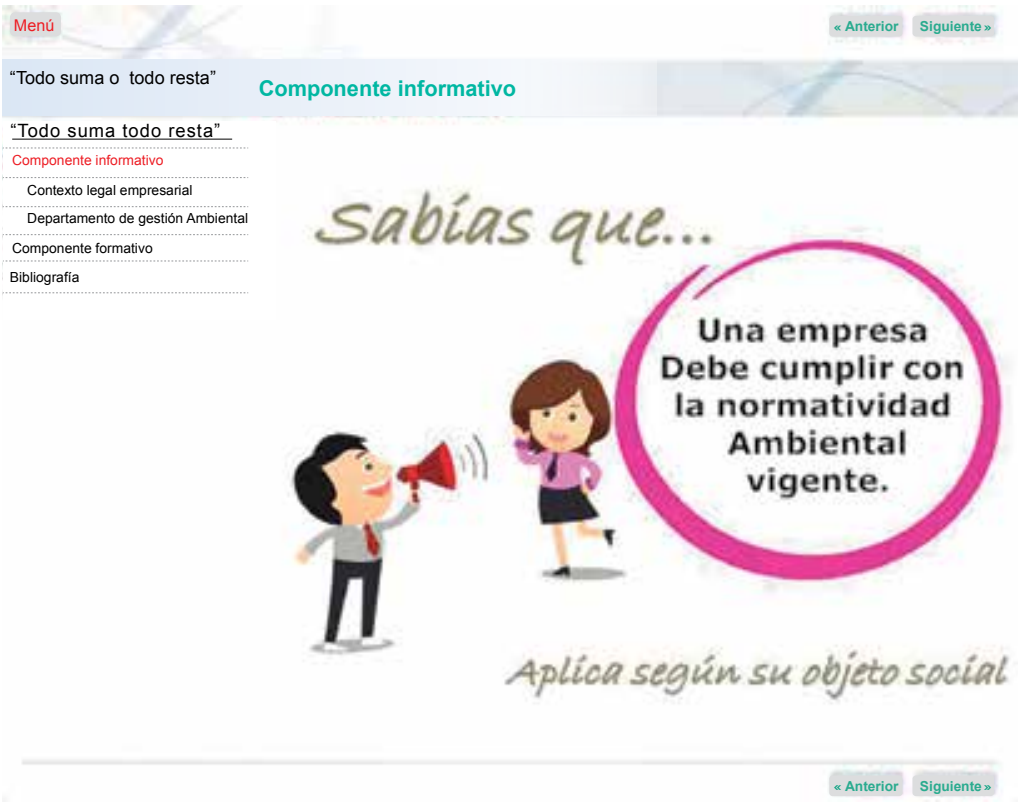

Diseño: Wilfredo Salinas.

Finalmente, se reconoce que una ventaja principal del objeto virtual de aprendizaje fue el nivel de usabilidad y la posibilidad de ponerlo en funcionamiento, tanto en plataformas web de libre acceso como en un archivo en formato html con acceso directo al recurso en el cual se integraron videos educativos y cuestionarios con preguntas que orientan a los usuarios en la práctica de comportamientos ambientales responsables.

Figura 7. Aspecto de la página de componente informativo del objeto de aprendizaje

"Todo suma o todo resta"

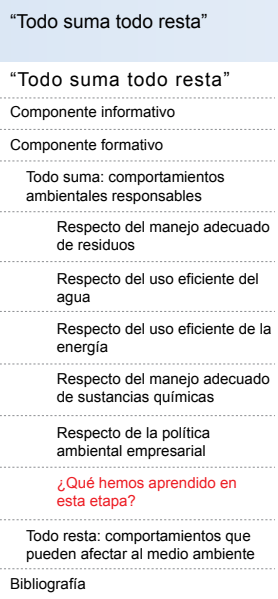

? Pregunta de Selección Múltiple

Dos respuestas son asertivas. ¿Cuáles son?

La manipulación de una sutancia quimica debe hacerse con los elementos de protección personla pertinentes. esta información se encuentra de forma veraz en :

$\square$ Hojas datos de seguridad - MSDS de la sustancia quimica

$\square$ Compañeros de trabajo

$\square$ Etiqueta de la sustancia quimica

Fuente: Diseño Wilfredo Salinas. 
Figura 8. Aspecto del componente formativo del objeto de aprendizaje "Todo suma o todo resta"

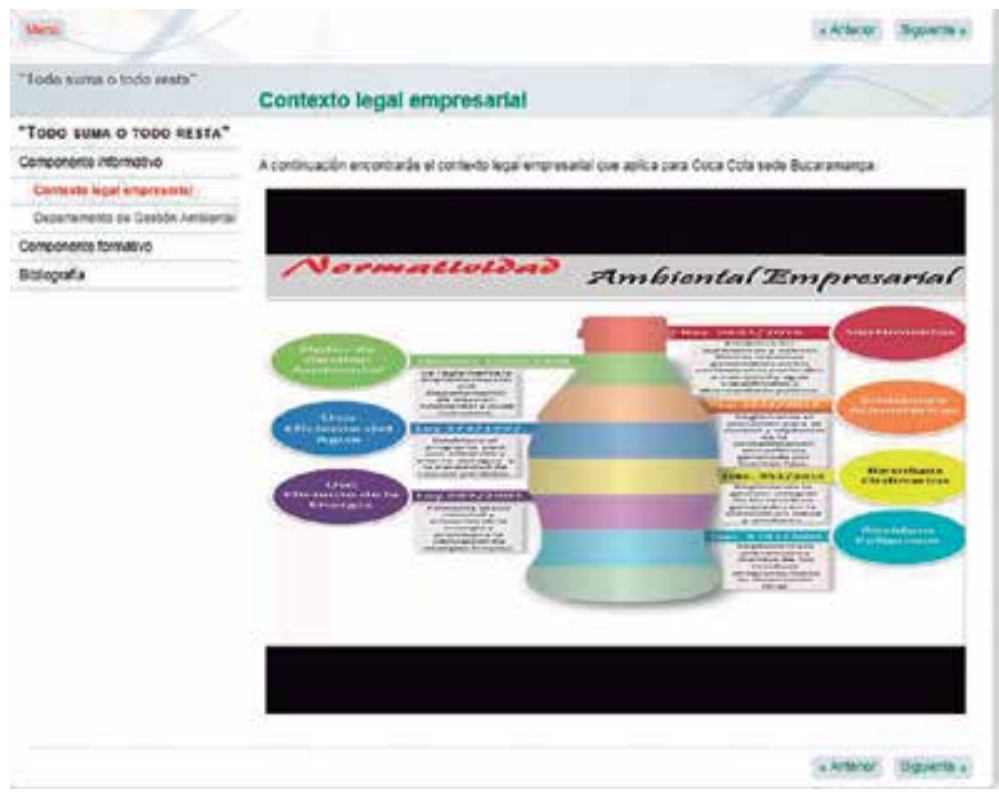

Fuente: Diseño Wilfredo Salinas.

\section{Referencias}

Álvarez, P., \& Vega, P. (2009). Actitudes ambientales y conductas sostenibles. Implicaciones para la educación ambiental. Revista de Psicodidáctica, 14, 245-260.

Barbas, C.A. (2012). Educomunicación: Desarrollo, enfoques y desafíos en un mundo interconectado. Foro de educación, 14, 157-175.

Bonilla, C. E., \& Rodríguez, P. (2010). Más allá del dilema de los métodos, la investigación en ciencias sociales. Consultado el 15 de mayo de 2015 [Libro en línea]. Recuperado de https://es.scribd.com/doc/26062421/Mas-alla-del-dilema-de-los-metodos

Correa, S., Puerta, A., \& Restrepo, B. (2002). Investigación evaluativa. Programa de especialización teoría, métodos y técnicas de investigación social. Bogotá. ARFO Editores e Impresores Ltda.

Decreto 1299 del 22 de abril de 2008. Ministerio de Ambiente, Vivienda y Desarrollo Territorial, Bogotá, D.C., Colombia.

Martínez, C. P. (2006). El método de estudio de caso. Estrategia metodológica de la investigación científica. Barranquilla: Pensamiento \& Gestión.

Ramírez, L., Arcila, A., Buriticá, L., \& Castrillón, J. (2004). Paradigmas y modelos de investigación, (2), 1-126. Fundación Universitaria Luis Amigó.

Santo Domingo, R.D. (2000). Los métodos y técnicas del modelo andragógico. Cancún: Universidad del Caribe. 